\title{
Modelo elástico simple para el estudio de pandeo en muros de hormigón armado
}

\section{Simple elastic model to study buckling in reinforced concrete walls}

Fecha de entrega: 10 de diciembre 2019

Fecha de aceptación: 26 de marzo 2020

\section{Alejandro Morales y Wendy Wiegand}

Escuela de Ingeniería Civil, Universidad de Valparaíso, General Cruz 222, Valparaíso, Chile, alejandro.morales@uv.cl, wendy.wiegand@uv.cl

El objetivo de este trabajo es obtener una expresión simple, que permita determinar el espesor mínimo de muros de hormigón armado esbeltos para prevenir pandeo. La expresión se obtuvo a partir de la teoría de placas delgadas, con un análisis lineal elástico, considerando un enfoque energético y el método de Ritz. El modelo propuesto considera sólo la zona comprimida del muro, con una distribución de carga axial, proveniente de demandas de flexo-compresión, consistente con las tensiones de compresión en el hormigón a lo largo de la distancia medida desde la fibra extrema en compresión al eje neutro. Los resultados obtenidos presentan razonable coincidencia cualitativa con lo observado en laboratorios, sin embargo, resultan ser poco conservadores en términos cuantitativos.

Palabras claves: pandeo, muros, hormigón armado, espesor mínimo
This work aims to obtain a simple expression, which allows defining a minimum thickness for reinforced concrete slender walls in order to avoid buckling. This expression was obtained based on the thin plates theory, with a linear-elastic analysis, considering an energetic approach and the Ritz method. The proposed model takes account for only the compressed zone of the wall, with an axial load distribution obtained from the flexocompression demands, in other words, the compression stresses in the concrete along the distance between the extreme fiber in compression and the neutral axis. The results obtained present an adequate qualitative agreement with the experimental observations, however, quantitatively the results are non-conservative.

Keywords: buckling, walls, reinforced concrete, minimum thickness

\section{Introducción}

Es un hecho que en los últimos años los avances en las normas y códigos de diseño sismorresistente han contribuido a mejorar la calidad de las estructuras nuevas, particularmente la aplicación de las reglas de diseño por capacidad, que minimizaron las clásicas fallas por corte en muros de hormigón armado (Sritharan et al., 2014). Sin embargo, a pesar de estos avances, observaciones de campo realizadas luego de la ocurrencia de los terremotos del año 2010 en Chile $\left(M_{\mathrm{w}}=8.8\right)$ y los terremotos en Canterbury en los años 2010 y $2011\left(M_{\mathrm{w}}=7.1, M_{\mathrm{w}}=7.7\right.$, respectivamente), han mostrado un patrón recurrente $\mathrm{y}$ poco común de falla asociado a inestabilidad fuera del plano o pandeo en el borde de muros (Sritharan et al., 2014; Rosso et al., 2016), como se puede ver en la Figura 1. Una consecuencia de este tipo de falla es que en muros, donde se adoptaron detallamientos especiales de borde que otorgan ductilidad, la "rótula plástica" esperada no se desarrolló; en otras palabras, estos muros no alcanzaron su máxima capacidad de deformación ni resistencia, fallando prematuramente por pandeo.
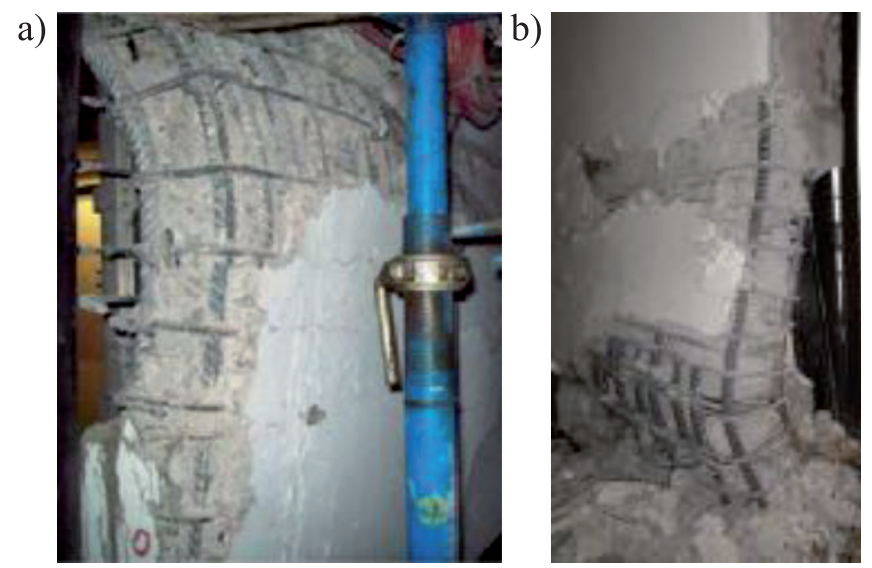

Figura 1: Ejemplos de pandeo o inestabilidad fuera del plano: a) terremoto de Chile, año 2010 (Wallace et al., 2012) y b) terremoto de Nueva Zelanda año 2011 (Kam et al., 2011) 
En cuanto a la práctica de diseño, los muros de hormigón armado son diseñados para tener suficiente resistencia y adecuada capacidad de deformación (ductilidad flexural), previniendo fallas frágiles debidas a corte, anclaje inadecuado del refuerzo, insuficiente longitud de traslapo de armaduras y deslizamiento del muro en su interfaz con la fundación. Algunos códigos de diseño buscan una respuesta dúctil de los muros a través de la filosofía de diseño por capacidad, e.g. NZS3101 (2006) y Eurocode 8 (2004), otros persiguen el mismo objetivo buscando un comportamiento dúctil sin usar esta filosofía explícitamente (ACI318, 2014; DS60, 2011). Una característica común de los muros que resisten grandes momentos y cortes es que son diseñados con elementos de borde, estos corresponden a regiones ubicadas en los extremos de los muros (bordes) con refuerzo adicional, mayor espesor o ambos. Haciendo una analogía con columnas de hormigón armado, estas regiones usan una combinación de alta concentración de refuerzo (longitudinal y transversal) para tolerar las altas demandas de deformación por compresión, necesaria para que el muro desarrolle una respuesta dúctil.

Adicionalmente, para limitar la falla prematura por pandeo fuera del plano en la zonas de potencial rótula plástica (sección crítica de los muros), lo común es definir espesores mínimos para los muros de hormigón armado. La NZS3101 (2006), por ejemplo, controla el mínimo espesor de los muros en función de la longitud de los mismos y su relación de aspecto. El Eurocode 8 (2004) y ACI318 (2014), en cambio, limitan el espesor de los elementos de borde con criterios que no se basan en limitar el potencial pandeo del muro producto de grandes deformaciones en la armadura longitudinal (Sritharan et al., 2014).

En este trabajo se propone, a través de un análisis elástico, determinar el mínimo espesor requerido para que un muro delgado de hormigón armado no pandee bajo la acción de cargas que provocan una deformación unitaria última del $0.3 \%$ en el hormigón. El análisis se fundamenta en la ecuación de placa (1), que representa el comportamiento lineal elástico de una placa delgada, como la mostrada en la Figura 2. La ecuación (1) se determinó bajo ciertos supuestos o "hipótesis de Kirchhoff", presentados a continuación: $i$ ) material elástico, homogéneo e isotrópico, ii) placa inicialmente plana, iii) espesor de placa pequeño en comparación con otras dimensiones de la placa, iv) se desarrollan pequeñas deformaciones, $v$ ) secciones planas permanecen planas, $v i$ ) las deflexiones son perpendiculares al plano inicial, vii) esfuerzos perpendiculares al plano medio son despreciables y viii) las deformaciones en la superficie media de la placa producidas por fuerzas en el plano de la misma son despreciables frente a deformaciones por flexión.

$$
\begin{aligned}
& \frac{\partial^{4} w}{\partial x^{4}}+2 \frac{\partial^{4} w}{\partial x^{2} \partial y^{2}}+\frac{\partial^{4} w}{\partial y^{4}}=\frac{N_{x}}{D} \frac{\partial^{2} w}{\partial x^{2}} \\
& D=\frac{E b_{w}{ }^{3}}{12(1-v)}
\end{aligned}
$$

En la ecuación (1) $w$ representa la deflexión del muro en la dirección $z, N_{x}$ las fuerzas actuando en el plano de la placa y $D$ corresponde a la rigidez flexural de la placa y está dada por (2). Por otro lado, en (2) $E$ es el módulo de elasticidad del material, $b_{w}$ es el espesor de la placa y $v$ el módulo de Poisson del material.

Nótese que en este análisis no se consideran cargas fuera del plano, asumiendo que el pandeo es producido por fuerzas internas actuando en el eje plano (ver Figura 2). Estás fuerzas $N_{x}$ son el resultado del comportamiento en flexo-compresión del muro.



Figura 2: Caracterización geométrica de la placa y fuerzas actuantes

Resulta claro que el problema de inestabilidad fuera del plano es inherentemente no lineal, por lo mismo, los resultados presentados en este trabajo son preliminares y deben complementarse con factores de corrección que permitan representar el fenómeno de la manera adecuada. En una segunda etapa de este estudio se trabajará en estos factores, a partir de resultados experimentales y/o análisis con elementos finitos no lineales. 


\section{Mecanismo que describe pandeo o falla fuera del plano}

Paulay y Goodsir (1985) fueron los primeros en estudiar, analizar y describir el mecanismo de falla fuera del plano (o pandeo) en muros delgados de hormigón armado, entendiéndose un muro delgado como aquel en que su espesor es pequeño en comparación a la altura del mismo, lo que lo hace susceptible a inestabilidad fuera del plano. Tomando como referencia la Figura 3, el mecanismo citado puede resumirse como sigue (Paulay y Priestley, 1993):

i) Con grandes demandas de desplazamiento (o curvatura), la región del borde inferior del muro está sometida a grandes tracciones que causan grietas anchas, prácticamente horizontales, a lo largo del muro. Con esto el refuerzo fluye, alcanzando posteriormente el endurecimiento por deformación.

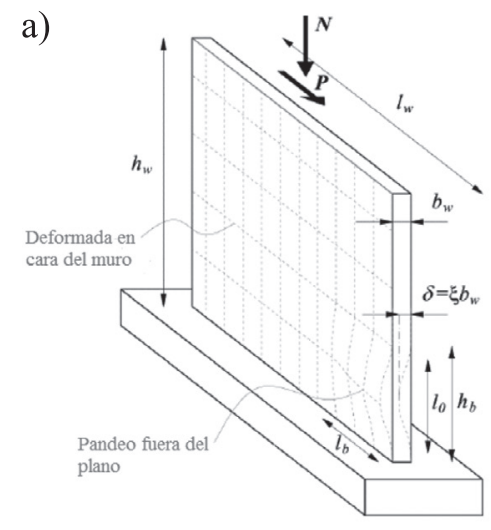

b)

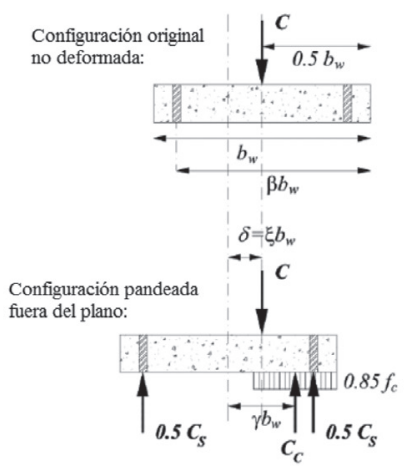

Figura 3: a) Caracterización geométrica del muro y b) equilibrio de fuerzas externas e internas a media altura de la región de pandeo (adaptada de Almeida et al., 2014)

ii) Con la descarga se recupera la deformación elástica, sin embargo, las grietas permanecen abiertas debido a las deformaciones por tracción inelástica acumuladas en las barras de refuerzo. Durante la recarga, y previo al cierre de las grietas, la fuerza de compresión debe ser resistida únicamente por las dos capas de refuerzo longitudinal (vertical). En esta etapa puede presentarse un incipiente desplazamiento fuera del plano, debido a imperfecciones constructivas y/o excentricidad de la fuerza axial $C$ que actúa en esta zona, ver Figura $3 b$.

iii) Mientras las barras de refuerzo logren mantener su rigidez axial antes de fluir en compresión, el desplazamiento fuera del plano tiende a permanecer pequeño. Sin embargo, en la medida que la compresión aumenta en el refuerzo longitudinal del lado cóncavo del muro, este puede fluir generando una abrupta reducción de la rigidez y un aumento de la deformación fuera del plano. En este punto, la capa de refuerzo del lado convexo, que aún no ha fluido, es la principal fuente de rigidez fuera del plano (Paulay y Priestley, 1993).

Dependiendo de la magnitud de la tracción (antes de la recarga), diversos escenarios pueden darse en la medida que avanza la compresión. Las grietas pueden cerrarse, en este caso la transferencia de fuerza de compresión se da a través del hormigón; si las grietas permanecen abiertas, la compresión se transfiere a través de la segunda capa de refuerzo. Considerando el último caso, los desplazamientos fuera del plano aumentarán violentamente, provocando el pandeo del muro.

Basado en este mecanismo, la máxima tracción inelástica en el borde de los muros se ha adoptado como indicador de la inestabilidad lateral de estos (Paulay y Priestley, 1993). Además, se ha observado que, con un incremento en el espesor del muro, el cerrado de las grietas puede ocurrir más temprano y se requieren mayores deformaciones por tracción inelástica para provocar pandeo (Dashti et al., 2018); este fenómeno se ilustra en la Figura 4. La Figura 4a muestra que debe existir un valor crítico de deformación unitaria en tracción inelástica para un espesor de muro dado $\left(t=b_{w}\right)$ que propicie el desarrollo de inestabilidad fuera del plano. Para evitar esto se debe aumentar el espesor del muro, provocando el cierre de grietas en un lado del mismo y, de esta manera, activar la contribución del hormigón a la capacidad de carga del muro (ver Figura $4 b$ ); o bien, evitar que la tensión en tracción desarrollada en el refuerzo alcance el valor crítico (ver Figura 4c).

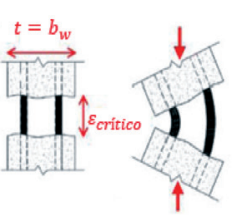

a)

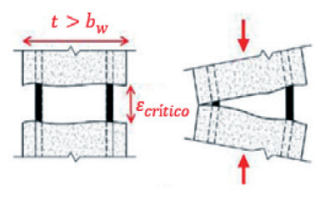

b)

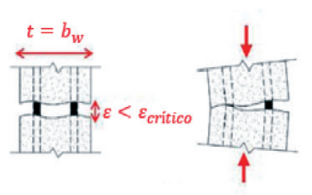

c)
Figura 4: Efectos de la deformación unitaria residual y el espesor del muro en el desarrollo de pandeo (Dashti et al., 2018) 


\section{Pandeo de placas delgadas: método energético}

Aplicar un enfoque energético para resolver el problema de pandeo de placas delgadas resulta práctico cuando la geometría de las placas es compleja y/o las condiciones de borde son variadas. En estos casos, resolver la ecuación diferencial (1) con un método basado en equilibrio puede resultar en un problema prácticamente imposible de resolver (Ventsel y Krauthammer, 2001). Considerando un enfoque energético, el equilibrio de un sistema será: estable, si la energía potencial de este estado tiene un valor mínimo comparado con valores correspondientes a otros posibles estados cercanos al estado de equilibrio; inestable, si la energía potencial es máxima y neutral si la energía no es máxima ni mínima.

Básicamente, al aplicar un criterio basado en energía potencial para un análisis de pandeo, se consideran dos posibles estados de la placa. El primero, $\Pi_{0}$, es el estado inicial de equilibrio bajo la acción de cargas en el eje del plano de placa, donde la superficie media permanece plana. El segundo, П, es un estado donde la superficie media está levemente curvada debido a pequeños desplazamientos virtuales aplicados a la placa. El equilibrio será estable si para todas las posibles pequeñas deflexiones $\Pi_{0}<\Pi$, inestable si $\Pi_{0}>\Pi$ y neutral si $\Pi_{0}=\Pi$.

El incremento en la energía potencial de deformación total en la superficie media de la placa, $\Delta \Pi$, está dado por la ecuación (3). Para la derivación de esta ecuación se asume que las tensiones resultantes son producto exclusivamente de las cargas en el borde externo del plano de la placa, además estas cargas no cambian debido a la deflexión producida por el pandeo.

$$
\Delta \Pi=\Pi-\Pi_{0}=\Delta U_{0}+U_{b}+\Delta \Omega_{\Gamma}
$$

En (3), $\Delta U_{0}$ es el incremento en la energía de deformación de la superficie media de la placa en pandeo, $U_{b}$ es la energía de deformación de la flexión y/o torsión en la placa y $\Delta \Omega_{\Gamma}$ es el incremento en el potencial de las fuerzas externas actuando en el plano de la placa.

La expresión para $\Delta U_{0}$ se muestra en la ecuación (4). El término $W_{e}$ corresponde al trabajo realizado por las fuerzas externas actuando en el plano, aplicadas en la superficie media de la placa.
El incremento en el potencial de las fuerzas externas actuando en el plano es igual al valor negativo del trabajo realizado por las fuerzas externas, es decir, $\Delta \Omega_{\Gamma}=W_{e}$.

La energía de deformación debido a flexión y torsión de una placa, $U_{b}$, está dada por la ecuación (5). Por lo tanto, el incremento en la energía potencial total de la placa en pandeo, $\Delta \Pi$, se obtiene sumando los términos mostrados en las ecuaciones (4) y (5).

$$
\begin{array}{r}
\Delta U_{0}=W_{e}+\frac{1}{2} \iint_{A}\left[N_{x}\left(\frac{\partial w}{\partial x}\right)^{2}+N_{y}\left(\frac{\partial w}{\partial y}\right)^{2}+2 N_{x y}\left(\frac{\partial w}{\partial x} \frac{\partial w}{\partial y}\right)\right] d A \\
U_{b}=\frac{1}{2} \iint_{A} D\left\{\left(\frac{\partial^{2} w}{\partial x^{2}}+\frac{\partial^{2} w}{\partial y^{2}}\right)^{2}-2(1-v)\left[\frac{\partial^{2} w}{\partial x^{2}} \frac{\partial^{2} w}{\partial y^{2}}-\left(\frac{\partial^{2} w}{\partial x \partial y}\right)^{2}\right]\right\} d A \\
\Delta \Pi=\frac{1}{2} \iint_{A} D\left\{\left(\frac{\partial^{2} w}{\partial x^{2}}+\frac{\partial^{2} w}{\partial y^{2}}\right)^{2}-2(1-v)\left[\frac{\partial^{2} w}{\partial x^{2}} \frac{\partial^{2} w}{\partial y^{2}}-\left(\frac{\partial^{2} w}{\partial x \partial y}\right)^{2}\right]\right\} d A \\
+\frac{1}{2} \iint_{A}\left[N_{x}\left(\frac{\partial w}{\partial x}\right)^{2}+N_{y}\left(\frac{\partial w}{\partial y}\right)^{2}+2 N_{x y}\left(\frac{\partial w}{\partial x} \frac{\partial w}{\partial y}\right)\right] d A
\end{array}
$$

En este trabajo, para hallar la carga crítica de pandeo se utilizará un método directo, particularmente el método de Ritz. Este último se usa comúnmente como una aproximación a la solución exacta del problema (Ventsel y Krauthammer, 2001).

El método energético desarrollado por Ritz aplica el principio de energía potencial mínima. De acuerdo al método de Ritz, la deformada o deflexión de la superficie de la placa es aproximada por una serie de la forma mostrada en la ecuación (7):

$$
w(x, y)=\sum_{i=1}^{\infty} C_{i} f_{i}(x, y)
$$

donde $f_{i}(x, y)$ son funciones que satisfacen individualmente, al menos las condiciones de borde cinemáticas del problema; $C_{i}$ son constantes desconocidas que se determinan a partir del principio de energía potencial mínima.

Al introducir la expresión mostrada en (7) en (6) de incremento de energía potencial, se obtendrá esta última como función de las constantes desconocidas .

$$
\Delta \Pi=\Delta \Pi\left(C_{1}, C_{2}, \ldots\right)
$$

La solución al problema de pandeo, finalmente, es un problema clásico de cálculo diferencial de máximos y mínimos, como muestra la ecuación (9). 
$\frac{\partial \Delta \Pi}{\partial C_{1}}=0, \quad \frac{\partial \Delta \Pi}{\partial C_{2}}=0, \ldots \quad \frac{\partial \Delta \Pi}{\partial C_{i}}=0, \ldots$

La ecuación (9) es un sistema lineal de ecuaciones no homogéneas cuya solución (constantes $C_{i}$ ) al ser sustituida en (7) entrega la solución del problema. Evidentemente, la exactitud del método de Ritz depende de que tan buenas son las funciones $f_{i}(x, y)$ asumidas, con respecto a la solución real del problema.

\section{Carga axial de pandeo}

El método de Ritz fue aplicado con el objetivo de determinar la carga axial de pandeo en muros delgados de hormigón armado; aplicar esta metodología requiere ciertas hipótesis y/o simplificaciones, además del hecho de ser un análisis lineal elástico. La Figura 5a muestra un segmento de muro sometido a esfuerzos de flexo-compresión, en esta se destaca la zona $A-B-C-D$ que corresponde a la zona comprimida del muro limitada por la base del muro $(A-B)$, la losa del piso superior $(C-D)$, el eje neutro $(A-D)$ y el borde libre del muro $(B-C)$. La Figura $5 \mathrm{~b}$ muestra la zona comprimida del muro, donde puede notarse que se trata de la misma situación mostrada en la Figura 2, en este caso $N_{x}$ corresponde a la distribución de tensiones en el hormigón asociadas a una deformación unitaria en compresión $\varepsilon_{m}$ de en el borde libre del muro, típicamente el 0.3-0.4\%. Este valor resulta importante ya que está asociado a la falla del hormigón sin confinar, además, en este punto gran parte de la zona comprimida del muro perdió el recubrimiento y por ende rigidez. a)

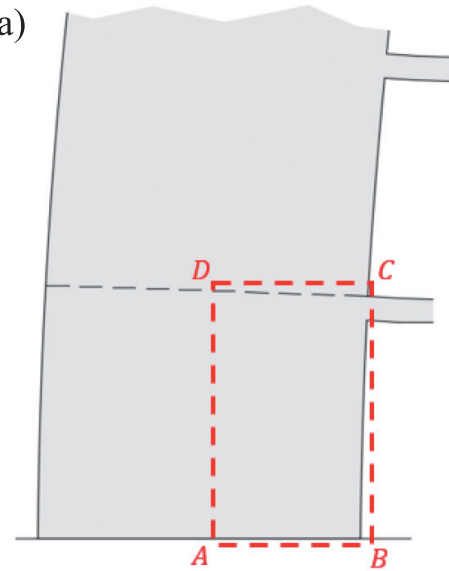

b)

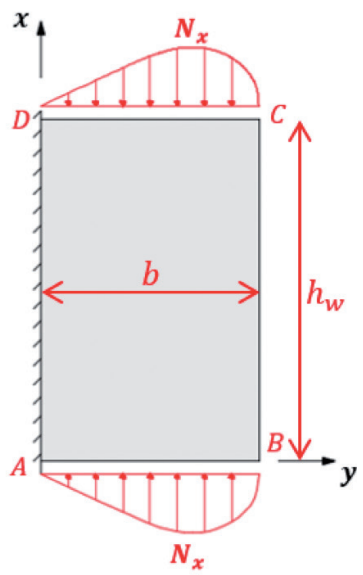

Figura 5: a) Problema original, muro en flexo-compresión y b) modelo de análisis para aplicar teoría de placas en la zona comprimida del muro
En este caso se asume el campo de desplazamientos (fuera del plano) mostrado en la ecuación (10), considerando su primera aproximación correspondiente a $N=1$, es decir, una función sinusoidal ponderada por una función lineal.

$w(x, y)=\operatorname{sen}\left(\frac{n \pi x}{a}\right) \sum_{i=1}^{N} C_{i} y^{i}$

El campo de desplazamiento cumple, además, las condiciones de borde del problema mostrado en la Figura $5 \mathrm{~b}$ y descritas en las ecuaciones (11a), (11b) y (11c).

$$
\begin{aligned}
& w(x, 0)=0 \\
& w(0, y)=0 \\
& w\left(h_{w}, y\right)=0
\end{aligned}
$$

La definición de la función $N_{x}$ se realiza a partir de una curva conocida del comportamiento del hormigón (ver Figura 6a), para lo cual se propone aproximar esta última mediante el polinomio cúbico mostrado en la ecuación (12). Si bien es cierto existen muy buenas funciones que describen la ley constitutiva del hormigón (Kent y Park, 1971; Mander et al., 1988), en general corresponde a funciones definidas por tramos de difícil manejo algebraico. La función propuesta tiene la ventaja de ser continua (no definida por tramos) y versátil, ya que con cuatro puntos es posible describir cualquier distribución de tensiones en el hormigón. En otras palabras, el polinomio permite conocer la distribución de tensiones para distintos valores de $\varepsilon_{m}$ lo que facilita el estudio del problema de pandeo para diversos estados límites. Nótese que la ecuación (12) es una manera alternativa de representar una ley constitutiva conocida del hormigón, como ilustra la Figura 6(b) para el caso de la ley constitutiva de Mander et al. (1988).

$$
N_{x}=b_{w}\left[\alpha_{3}\left(\frac{\varepsilon_{m}}{b} y\right)^{3}+\alpha_{2}\left(\frac{\varepsilon_{m}}{b} y\right)^{2}+\alpha_{1}\left(\frac{\varepsilon_{m}}{b} y\right)\right]
$$

En la ecuación (12) $b_{w}$ corresponde al espesor del muro, los factores $\alpha_{1}, \alpha_{2}$ y $\alpha_{3}$ están dados por las ecuaciones (13a) (13b) y (13c).

$$
\begin{aligned}
& \alpha_{1}=\frac{18 f_{1}-9 f_{2}+2 f_{3}}{2 \varepsilon_{m}} \\
& \alpha_{2}=-\frac{9\left(5 f_{1}-4 f_{2}+f_{3}\right)}{2 \varepsilon_{m}^{2}} \\
& \alpha_{3}=\frac{9\left(3 f_{1}-3 f_{2}+f_{3}\right)}{2 \varepsilon_{m}^{3}}
\end{aligned}
$$


a)

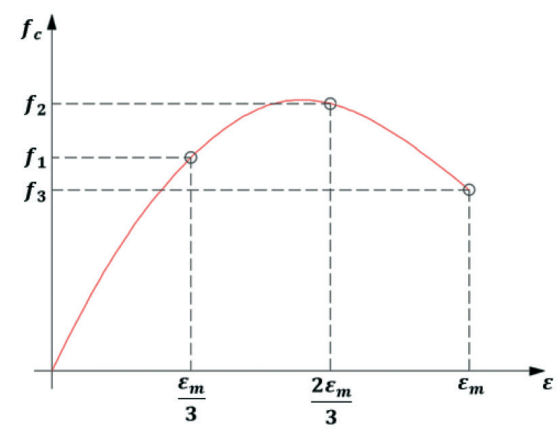

b)

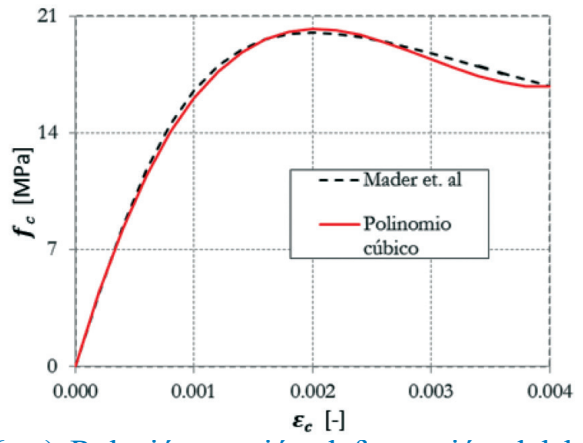

Figura 6: a) Relación tensión-deformación del hormigón y su definición a partir de un polinomio cúbico y b) ejemplo de ajuste

En un clásico problema de pandeo el objetivo es hallar la carga que lo genera. Sin embargo, en este caso se busca estimar la relación altura de entrepiso $\left(h_{w}\right)$ dividido en el espesor del muro $\left(b_{w}\right)$, asumiendo que la carga límite para que el muro pandee es la mostrada en (12). De esta manera, la idea es estimar el espesor mínimo requerido para que el muro no pandee antes de que el hormigón alcance su deformación unitaria última en compresión o la deformación que el diseñador estime. Reemplazando el campo de desplazamientos de (10) y la función de carga mostrada en (12) en (8) de incremento de la energía potencial, se tiene:

$$
\begin{aligned}
\Delta \Pi=\frac{b D \pi^{2}\left[b^{2} \pi^{2}-6 h_{w}^{2}(v-1)\right] C_{1}^{2}}{12 h_{w}^{3}} \\
-\frac{b^{3} \pi^{2} \varepsilon_{m}\left[15 \alpha_{1}+2 \varepsilon_{m}\left(6 \alpha_{2}+5 \varepsilon_{m} \alpha_{3}\right)\right] C_{1}^{2}}{240 h_{w}}
\end{aligned}
$$

Optimizando la ecuación (14), de acuerdo a lo definido en (9), se obtiene la ecuación (15):

$$
\begin{aligned}
\frac{\partial \Delta \Pi}{\partial C_{1}}=\frac{b D \pi^{2}\left[b^{2} \pi^{2}-6 h_{w}^{2}(v-1)\right] C_{1}}{6 h_{w}^{3}} & \\
-\frac{b^{3} \pi^{2} \varepsilon_{m}\left[15 \alpha_{1}+2 \varepsilon_{m}\left(6 \alpha_{2}+5 \varepsilon_{m} \alpha_{3}\right)\right] C_{1}}{120 h_{w}} & =0
\end{aligned}
$$

La ecuación (16) muestra el resultado obtenido para $\left(h_{w} /\right.$ $b_{w}$ ) y la Figura 7 a una representación gráfica de un caso particular del mismo, considerando un hormigón calidad G20 con $\varepsilon_{m}=0.3 \%$. La Figura $7 \mathrm{~b}$ ilustra cualitativamente la deformada adoptada por un muro en la condición de pandeo, esta deformación se estimó con (10). La Figura $7 \mathrm{~b}$ muestra, además, que la deformación se concentra en una longitud $h_{w}$ que corresponde a la altura de entrepiso del primer piso, a lo largo de la zona comprimida del muro de longitud $b$ (profundidad del eje neutro). Nótese que, como era esperable, la máxima deformación fuera del plano ocurre en el borde libre del muro y se ubica en el punto medio de la altura de entrepiso, este último punto difiere con los resultados obtenidos experimentalmente y con modelaciones numéricas más robustas, tal como se mostrará más adelante. Es importante destacar que, durante el desarrollo que condujo a la expresión mostrada en la ecuación (16), se impuso la condición $C_{1} \neq 0$ (ver ecuación (15)) garantizando que la expresión hallada no corresponde a la solución trivial o $w(x, y)=0$.

$$
\left(\frac{h_{w}}{b_{w}}\right)^{2}=\frac{20 \pi^{2} E}{12 \varepsilon_{m}\left(15 \alpha_{1}+12 \varepsilon_{m} \alpha_{2}+10 \varepsilon_{m}^{2} \alpha_{3}\right)} \cdot \frac{\left(\frac{b_{w}{ }^{2}}{h_{w}{ }^{2}}+6 \frac{(1-v)}{\pi^{2}}\right)}{\frac{b_{w}{ }^{2}}{h_{w}{ }^{2}}(1-v)}
$$

a)

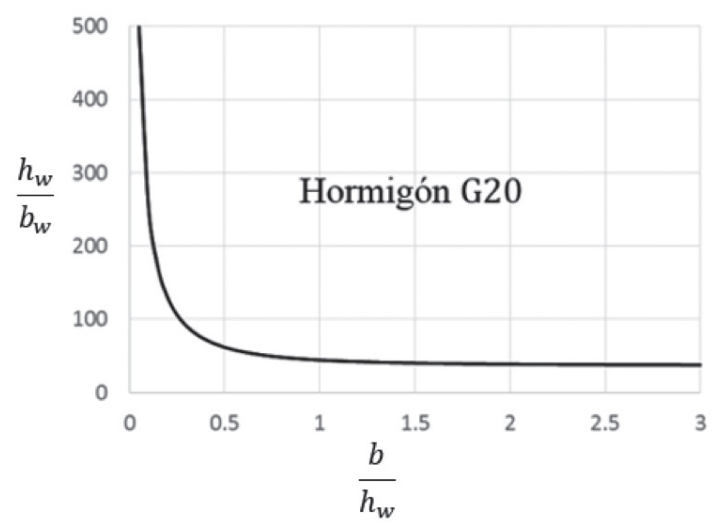

b)

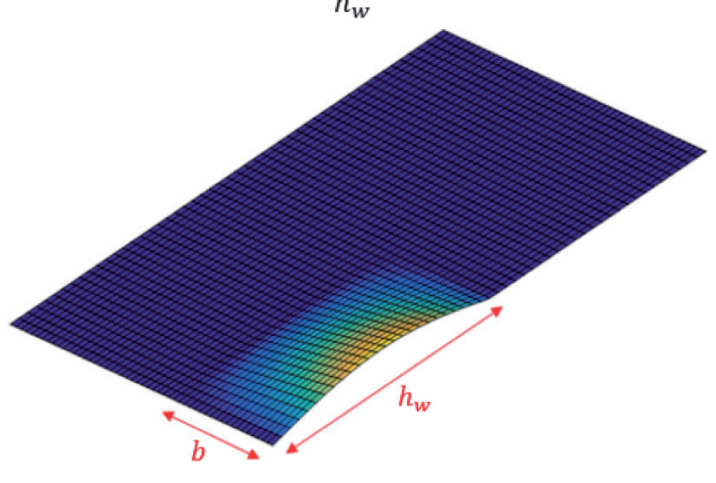

Figura 7: a) Relación $\left(h_{w} / b_{w}\right)$ crítico para un muro de hormigón armado de calidad G20 y b) deformada cualitativa del muro bajo la acción de pandeo 
La relación $\left(h_{w} / b_{w}\right)$ límite mostrada en la Figura 7a es del orden de 37 para valores de $\left(h_{w} / b_{w}\right)$ mayores que 1.5 , este número resulta poco conservador, alejándose de lo medido en laboratorios donde se reportan valores cercanos a 25 (Rosso et al., 2016) y lo comúnmente especificado en códigos de diseño. Por ejemplo, el ACI318 (2014) y el DS60 (2011) definen un valor de 16. Resultados previos obtenidos, usando una versión simplificada de la metodología propuesta, también mostraron ser poco conservadores (Wiegand y Morales, 2019). Sin embargo, este resultado es esperable ya que, como se manifestó previamente, la metodología propuesta se basa en un análisis lineal elástico y el fenómeno de estabilidad fuera del plano es un fenómeno no lineal.

\section{Limitaciones de la metodología propuesta e investigación futura}

Con respecto al fenómeno de inestabilidad fuera del plano (o pandeo), documentado y evaluado experimentalmente, la metodología propuesta presenta dos diferencias sustanciales. Primero, la rigidez flexural del muro; el nivel de fisuración previo al pandeo del muro reduce considerablemente la rigidez del mismo, este efecto es mucho mayor en la zona donde ocurre el pandeo ya que, básicamente, la rigidez es proporcionada por las barras de refuerzo longitudinal (ver Figura 4). Segundo, la ubicación de la zona de pandeo; el modelo propuesto ubica la máxima deformación fuera del plano en el centro de la altura de entrepiso $h_{w}$ (ver Figura 7b). Sin embargo, la evidencia experimental (ver Figuras 1 y 8a) y numérica, con elementos finitos no lineales (EFNL), muestra que la ubicación de la máxima deformación lateral (fuera del plano) se da en la parte baja del muro, tal como se puede ver en la Figura 8. La Figura 8a muestra el desplazamiento lateral del muro $\delta_{\text {oop }}$ y el desplazamiento lateral normalizado $\xi_{\text {oop }}\left(\delta_{\text {oop }} /\right.$ $b_{w}$ ), medido para distintos estados de carga (Rosso et al., 2016). La Figura 8b, por otro lado, muestra los resultados analíticos estimados con EFNL y su comparación con mediciones en laboratorio (Dashti et al., 2018).

Los resultados obtenidos en este trabajo, como se ha planteado, difieren de lo medido en laboratorios, mostrando discrepancias cuantitativas y/o cualitativas. Sin embargo, estas diferencias no son radicales, más aún si se considera que los resultados se obtuvieron de un análisis lineal elástico asumiendo una deformada lateral (o campo de


Figura 8: Deformación lateral fuera del plano, a) resultados experimentales (Rosso et al., 2016) y b) resultados numéricos usando EFNL (Dashti et al., 2018)

desplazamiento). Tomando estos resultados como punto de partida, se vislumbra como futura materia de investigación la inclusión de factores de corrección para la expresión mostrada en la ecuación (16) que consideren, en principio, modificar la rigidez flexural del muro y la ubicación de la zona de pandeo. Los factores se trabajarán a partir de resultados experimentales disponibles y de modelación usando Elementos Finitos No Lineales.

\section{Conclusiones}

En este trabajo se presentó una metodología simple para estimar el espesor mínimo requerido para prevenir el pandeo de un muro esbelto de hormigón armado, bajo la acción de cargas axiales provenientes de demandas de flexo-compresión. La metodología se fundamenta en el uso de la teoría de placas y la aplicación de un método energético, el método de Ritz. El análisis es lineal elástico y trabaja sólo con la zona comprimida del muro, entiéndase un ancho igual a la profundidad del eje neutro, la cual es cargada con una distribución de fuerzas consistente con la desarrollada en el hormigón. Además, se asume un campo de desplazamiento o deformada límite por pandeo. El resultado es una expresión para determinar la relación límite entre la altura de entrepiso y el espesor del muro $\left(h_{w} / b_{w}\right)$ como función de la calidad del hormigón, sus propiedades físicas $(E, v)$, la altura de entrepiso $\left(h_{w}\right)$ y la profundidad el eje neutro $\left(b_{w}\right)$. Los resultados obtenidos son poco conservadores, pero no radicalmente lejanos a los medidos en laboratorio. Las diferencias son, básicamente, la longitud o zona de pandeo del muro y la relación $\left(h_{w} / b_{w}\right)$ para la cual se produce la inestabilidad. Una segunda etapa de este trabajo se orientará en la búsqueda de factores de ajuste que incluyan la no-linealidad del fenómeno en la expresión propuesta, estos factores estarán asociados a 
cuantificar el nivel de agrietamiento del muro y a modificar el campo de desplazamiento lateral $w(x, y)$. La metodología propuesta resulta útil y de fácil aplicación ya que la información que requiere es esencialmente la misma que el diseñador utiliza, por ejemplo, para el cálculo y estimación del confinamiento en muros, reduciéndose a un análisis seccional (diagrama momento-curvatura, estimación de profundidad neutra y deformación unitaria del hormigón).

\section{Referencias}

ACI318 (2014). Building code requirements for structural concrete and commentary. American Concrete Institute, Farmington Hills, USA

Almeida, J., Rosso, A., Beyer, K. and Sritharan, S. (2014). New experimental findings on the stability of thin reinforced concrete walls. 5as Jornadas Portuguesas de Engenharia de Estruturas, Lisboa, Portugal

Eurocode 8 (2004). Design provisions for earthquake resistance of structures - Part 1: General rules, seismic actions and rules for buildings. European Committee for Standardization, Brussels, Belgium

Dashti, F., Dhakal, P. and Pampanin, S. (2018). Validation of a numerical model for prediction of out-of-plane instability in ductile structural walls under concentric in-plane cyclic loading. Journal of Structural Engineering 144(6), 04018039

DS60 (2011). Reglamento que fija los requisitos de diseño y cálculo para el hormigón armado y deroga el decreto $\mathrm{N}^{\circ} 118$, de 2010. Decreto Supremo, Ministerio de Vivienda y Urbanismo, Diario Oficial de la República de Chile, № 40.133, 1-8

Kam, W.Y., Pampanin, S. and Elwood, K. (2011). Seismic performance of reinforced concrete buildings in the 22 February Christchurch (Lyttelton) earthquake. Bulletin of the New Zealand Society for Earthquake Engineering 44(4), 239-278
Kent, D.C. and Park, R. (1971). Flexural members with confined concrete. Journal of the Structural Division 97(7), 1969-1990

Mander, J.B., Priestley, M.J.N. and Park, R. (1988). Theoretical stress-strain model for confined concrete. Journal of Structural Engineering 114(8), 1804-1825

NZS3101 (2006). Concrete structures standard, part 1: The design of concrete structures. Standards New Zealand, Wellington, New Zealand

Paulay, T. and Goodsir, W.J. (1985). The ductility of structural walls. Bulletin of the New Zealand Society for Earthquake Engineering 18(3), 250-269

Paulay, T. and Priestley, M.J.N. (1993). Stability of ductile structural walls. Structural Journal 90(4), 385-392

Rosso, A., Almeida, J.P. and Beyer, K. (2016). Stability of thin reinforced concrete walls under cyclic loads: state-of-the-art and new experimental findings. Bulletin of Earthquake Engineering 14(2), 455-484

Sritharan, S., Beyer, K., Henry, R.S., Chai, Y.H., Kowalsky, M. and Bull, D. (2014). Understanding poor seismic performance of concrete walls and design implications. Earthquake Spectra 30(1), 307-334

Ventsel, E. and Krauthammer, T. (2001). Thin plates and shells. Theory, analysis, and applications. Marcel Dekker, New York, USA

Wallace, J.W., Massone, L.M, Bonelli, P., Dragovich, J., Lagos, R., Lüders, C. and Moehle, J. (2012). Damage and implications for seismic design of RC structural wall buildings. Earthquake Spectra 28(S1), 281-299

Wiegand, W. y Morales, A. (2019). Pandeo elástico en muros esbeltos de hormigón armado. XII Congreso Chileno de Sismología e Ingeniería Sísmica ACHISINA, Valdivia, Chile, trabajo $\mathrm{N}^{\circ} 1487$ 